\title{
The axisymmetric frictional receding contact of a layer pressed against a half-space by pressure outside a disk
}

\author{
J.P. Lopes ${ }^{\mathrm{a}, *}$ D.A. Hills ${ }^{\mathrm{a}}$ \\ ${ }^{a}$ Department of Engineering Science, University of Oxford, \\ Parks Road, Oxford OX1 3PJ, United Kingdom
}

\begin{abstract}
This paper uses a distribution of ring dislocations to find the solution for the frictional axisymmetric receding contact of a semi-infinite layer pressed against a half-space by a uniform surface pressure exerted outside a disk. Three cases are considered: the contact is fully closed and stuck; the contact is fully closed and slipping; the contact is partially open and slipping. The tractions in the contact interface are obtained, as well as the self-determining points of closure and stick.
\end{abstract}

Keywords: Axisymmetric, Receding Contact, Ring dislocations

\section{Introduction}

The main characterization of contacts is done by comparing the extent of the contact in the deformed configuration with the initial configuration in the unloaded state. While the initial state is determined by the geometric features of the bodies and their fixtures, the deformed contact state generally depends on the nature of the applied loads, the level of loading and the elastic constants of the materials [1].

The classification is done by observing whether or not new surface points come in contact as the bodies deform. In advancing contacts, new surface points come in contact as the load is applied and, as a consequence, the deformed contact is not contained in the initial state. However, in receding contacts, the contact shrinks resulting in the deformed contact being contained in the initial state.

In machines or structures with carefully fitted parts, receding contacts are more likely to appear than advancing contacts, because of the opening of gaps between the individual parts as they distort under application of loads.

A property of some receding contacts is that the contact area in the deformed configuration is independent of the applied load and, consequently, the change between undeformed/deformed configurations is discontinuous, i.e. the contact 'snaps' to the deformed configuration upon applying a load $[1,2]$. This presents a challenge in obtaining solutions through the finite element method, as a large portion of the nodes change status from being in contact to being free with any increment of load.

One of the applications of receding contacts in industry is the analysis of frictional contacts in bolted joints, which

\footnotetext{
* Corresponding author

Email addresses: jhonatan.dapontelopes@eng.ox.ac.uk (J.P. Lopes), david.hills@eng.ox.ac.uk (D.A. Hills)
}

are naturally axisymmetric, either for the whole joint or for its individual elements. However, the scarce work currently present in the literature for receding contacts is focused either on plane contacts [3, 4] or on frictionless cases $[5,6,7]$.

This paper proposes the study of the frictional axisymmetric receding contact of a homogeneous semi-infinite layer of thickness $a$, Poisson's ratio $\nu$ and modulus of rigidity $\mu$, pressed against an elastically similar half-space $z \geq a$ (Figure 1a) by a semi-infinite patch of pressure applied to the layer's surface $(z=0)$, such that

$$
\begin{array}{lrl}
\sigma_{z z}(r, 0) & =-p, & r \geq b \\
\sigma_{z z}(r, 0) & =0, & 0 \leq r<b .
\end{array}
$$

Furthermore, the objective of this study is to find the solution to the proposed problem using ring dislocations to introduce corrections to the stresses in the contact interface as a distribution of strain nuclei. Initially, we assume that the contact is in a fully closed and stuck configuration. Then, dislocation densities are applied to correct the stresses when this condition is violated.

\section{Adhered Solution}

First, we assume that the unloaded region of radius $b$ is small enough to prevent contact opening and that the coefficient of friction $f$ is high enough to prevent slip throughout the contact area, as shown in Figure 1a (Case $I$ ). In this case, the two bodies behave as one, since the contact is closed and stuck everywhere. Therefore, the state of stress in the bodies is equivalent to a half-space $z \geq 0$ under the same loading. In the cylindrical coordinate set shown in the figure, the state of stress is given by the superposition 


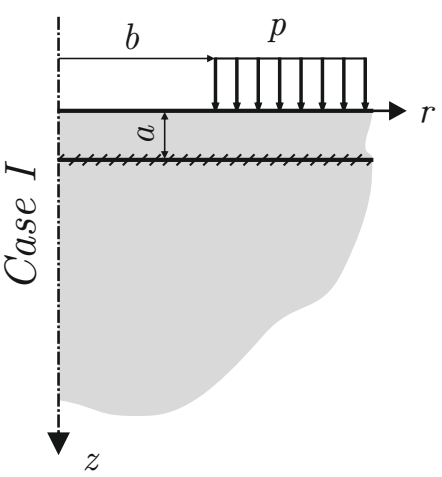

(a)

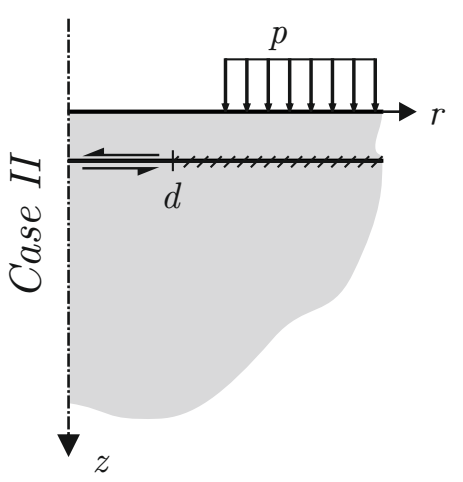

(b)

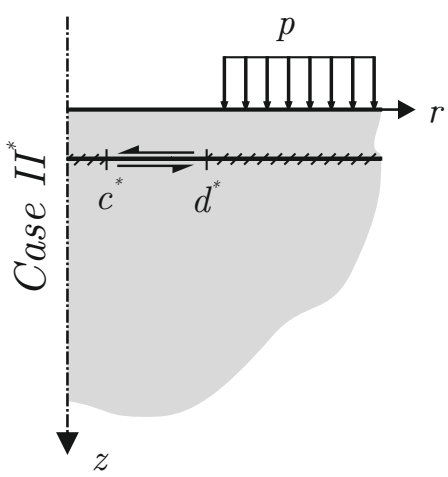

(c)

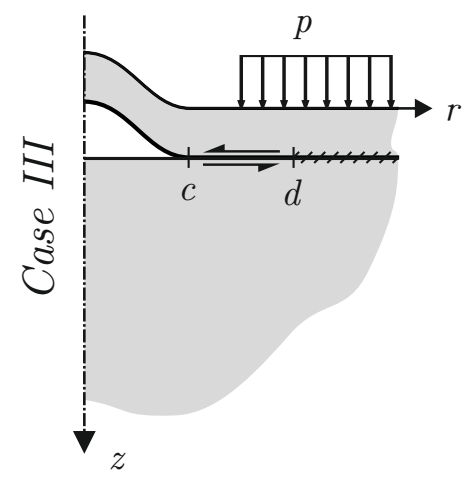

(d) of a uniform pressure $p$ throughout the surface of the halfspace and a circular patch of uniform normal traction $-p$ and radius $b[8,9,10]$. The tractions $\tilde{\sigma}_{i j}(r, z)$ arising on any radial cut ( $z=$ constant surface) are given by:

$$
\begin{aligned}
& \tilde{\sigma}_{z z}(r, z)=-p\left\{1+\frac{2 z}{\pi}\left[\frac{r^{2}+z^{2}-b^{2}}{\left(l_{2}\right)^{3}\left(1-k^{2}\right)} \boldsymbol{E}(k)+\right.\right. \\
& \left.\left.\frac{b^{2}-\left(l_{1}\right)^{2}}{\left(l_{2}\right)^{3}\left(1-k^{2}\right)} \boldsymbol{K}(k)-\frac{1}{l_{2}} \boldsymbol{\Pi}(n ; k)\right]\right\} \\
& \tilde{\sigma}_{r z}(r, z)=p\left\{\frac{2 z^{2}}{\pi}\left[\frac{\left(1+k^{2}\right) \boldsymbol{E}(k)-\left(1-k^{2}\right) \boldsymbol{K}(k)}{\left(k^{2}-1\right)^{2} l_{2} r}\right]\right\}
\end{aligned}
$$

where

$$
\begin{aligned}
& l_{1}=\frac{1}{2}\left\{\sqrt{(r+b)^{2}+z^{2}}-\sqrt{(r-b)^{2}+z^{2}}\right\} \\
& l_{2}=\frac{1}{2}\left\{\sqrt{(r+b)^{2}+z^{2}}+\sqrt{(r-b)^{2}+z^{2}}\right\} \\
& k=\frac{l_{1}}{l_{2}} \\
& n=\left(\frac{l_{1}}{r}\right)^{2}
\end{aligned}
$$

and $\boldsymbol{K}(k), \boldsymbol{E}(k), \boldsymbol{\Pi}(n ; k)$ are the complete elliptic integrals of the first, second and third kind respectively. These functions are continuous and bounded at every point inside the half-space, but $\Pi(n ; k)$ cannot be evaluated numerically along the $z$-axis, since $r=0$ there. In this region, we take the limits as $r \rightarrow 0$, giving:

$$
\begin{aligned}
& \tilde{\sigma}_{z z}(0, z)=-p\left\{\frac{z^{3}}{\left(b^{2}+z^{2}\right)^{3 / 2}}\right\} \\
& \tilde{\sigma}_{r z}(0, z)=0 .
\end{aligned}
$$

If the contact is, indeed, fully closed and adhered, the normal $N(r)$ and shear $S(r)$ tractions at the layer/halfspace interface are given by:

$$
\begin{aligned}
N(r) & =\tilde{\sigma}_{z z}(r, a) \\
S(r) & =\tilde{\sigma}_{r z}(r, a) .
\end{aligned}
$$

Figure 2 shows the normalised contact pressure and shear to normal stress ratio for the adhered solution. Analysing the stress ratio (Figure $2 \mathrm{~b}$ ), there is a minimum coefficient of friction that results in the contact being fully stuck for each $b / a$. When $b / a$ is relatively large, however, there is no realistic coefficient of friction that prevents slip. Thus, we expect that some pairs of $b / a$ and $f$ result in a case where the contact is closed but slipping from the axis 


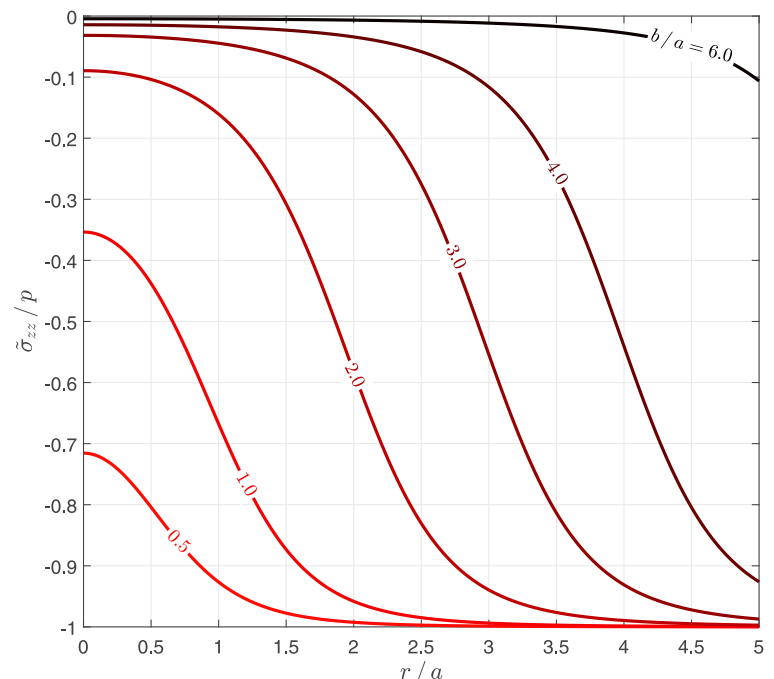

(a)

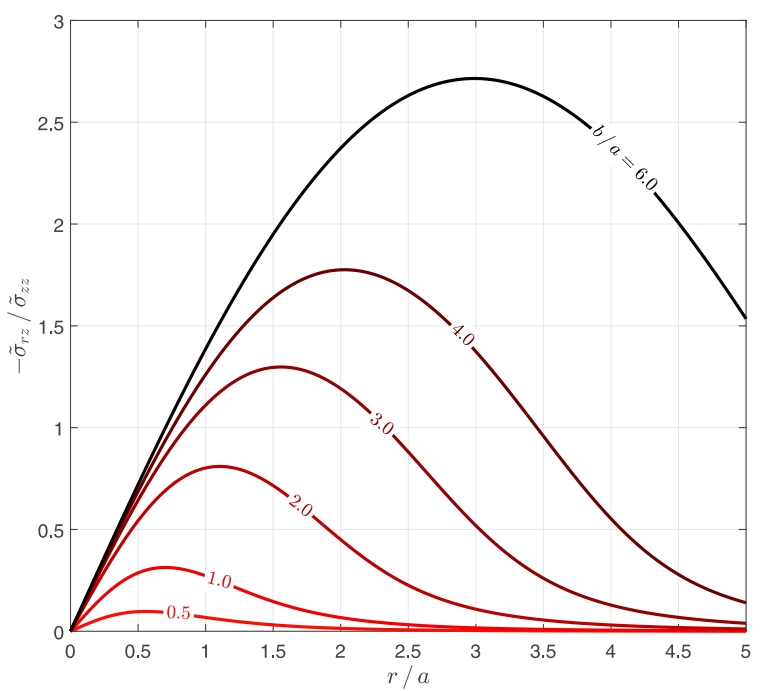

(b)

Figure 2: Normalised tractions for the adhered (bilateral) solution. (a) Normalised contact pressure. (b) Shear to normal stress ratio.

of symmetry to a self-determining radius $d$ (Case $I I$, Figure 1b). Analysing the contact pressure (Figure 2a), we expect the contact to be closed when the normalised radius of the unloaded disk $b / a$ is small. As the unloaded region widens, the contact pressure becomes zero at the interface, which results in the development of an open region. Therefore, for some pairs of $b / a$ and $f$, we expect the contact to be partially open from the axis of symmetry to a radius $c$ and closed and slipping from $c$ to $d$ (Case $I I I$ ).

\section{Formulation}

If the contact is not fully stuck and closed, the bilateral solution obtained in Section 2 is no longer valid. A mod- ified solution can be obtained by developing expressions for the tractions at the contact interface as the sum of the adhered (bilateral) solution together with a correction in the form of an integral representation of slip and opening as a distribution of climb and glide dislocations. The dislocations needed are all 'edge' in character, with their Burgers vector lying in a $\theta=$ constant plane. We emphasise that the introduction of dislocations is used solely as a mathematical device to introduce corrections in our formulation as a distribution of strain nuclei and do not imply the introduction of physical defects in the micro-structure of the material. A more detailed explanation can be found in [11].

First, we introduce a $b_{r}(\xi)$ glide dislocation loop of radius $\xi$, lying at a depth $a$. It may be formed by making a path cut along the disk $0 \leq r \leq \xi$ and sliding the surfaces by a constant amount $b_{r}$. This models contact slip and will induce the following tractions $\hat{\sigma}_{i z}(r)(i=r, z)$ along the same disk

$$
\hat{\sigma}_{i z}(r)=G_{i z}^{r}(r, \xi) b_{r}(\xi)
$$

The influence functions $G_{i z}^{r}(r, \xi)$ are functions of LipschitzHankel integrals and have extremely complicated forms. For a half-space, these are given by Paynter et al. [12] (see also $[13,14])$. They are bounded ('regular') when $i=z$ and display a Cauchy singularity when $i=r$.

Next, we introduce a $b_{z}(\xi)$ climb dislocation loop of radius $\xi$, lying at a depth $a$. Now, the dislocation loop may be formed by making a path cut along $0 \leq r \leq \xi$ and inserting a disk of thickness $b_{z}$. This models contact opening and will induce the following tractions $\hat{\sigma}_{i z}(r)(i=$ $r, z)$ along the same disk

$$
\hat{\sigma}_{i z}(r)=G_{i z}^{z}(r, \xi) b_{z}(\xi)
$$

Once again, the influence functions $G_{i z}^{z}(r, \xi)$ are functions of Lipschitz-Hankel integrals and have extremely complicated forms. For a half-space, these are also given by Paynter et al. [12]. This time, they are bounded ('regular') when $i=r$ and display a Cauchy singularity when $i=z$.

The resulting normal $N(r)$ and shear $S(r)$ tractions along the contact interface are given by

$$
\begin{aligned}
N(r)= & \tilde{\sigma}_{z z}(r)+\int_{\text {opening }} G_{z z}^{z}(r, \xi) B_{z}(\xi) \mathrm{d} \xi+ \\
& \int_{\text {slip }} G_{z z}^{r}(r, \xi) B_{r}(\xi) \mathrm{d} \xi \\
S(r)= & \tilde{\sigma}_{r z}(r)+\int_{\text {opening }} G_{r z}^{z}(r, \xi) B_{z}(\xi) \mathrm{d} \xi+ \\
& \int_{\text {slip }} G_{r z}^{r}(r, \xi) B_{r}(\xi) \mathrm{d} \xi
\end{aligned}
$$


where $B_{i}(\xi)=d b_{i} / d \xi, i=r, z$. Glide dislocations are installed over the whole of the length of the contact interface where slipping occurs, including the open portion, and climb dislocations are installed over the part of the contact interface which is open. Equations (15) and (16) form the basis of the solution and integral equations may be generated in slightly different forms for the cases $I I$ and $I I I$ of response described in Section 2.

\subsection{Case $I I$}

Case II represents the situation where there is a region of slip of radius $d$ but there is no separation. The coefficient of friction $f$ is small enough so that slip occurs but is high enough to prevent contact opening. The point $d$ where the contact sticks is self-determining and, thus, is an output of the problem.

In the slip region, the boundary conditions require that the normal stress must be compressive and the shear stress must be limited by friction:

$$
S(r)=-f N(r), \quad N(r)<0, \quad 0 \leq r \leq d .
$$

Since there are no open regions in Case $I I$, only glide dislocations $b_{r}$ are needed to correct the stresses. Applying eqs. (15) and (16) to eq. (17), we obtain the following Cauchy integral equation

$$
\begin{array}{rlrl}
\int_{0}^{d}[ & \left.G_{r z}^{r}(r, \xi)+f G_{z z}^{r}(r, \xi)\right] B_{r}(\xi) \mathrm{d} \xi & = & \\
- & {\left[\tilde{\sigma}_{r z}(r)+f \tilde{\sigma}_{z z}(r)\right]} & 0 \leq r \leq d
\end{array}
$$

The problem consists now in finding the dislocation density $B_{r}(\xi)$ that satisfies eq. (18). Since the functions present in eq. (18) are of extreme complexity, there is no hope of analytically inverting the singular integral equation. It must be solved numerically using a standard numerical quadrature devised by Erdogan, Gupta and Cook [15]. First, we put them in standard form over the intervals $[-1,1]$ utilizing the substitutions

$$
s=\frac{2 \xi}{d}-1, \quad t=\frac{2 r}{d}-1 \quad 0 \leq r, \xi \leq d
$$

which gives

$$
\begin{aligned}
\int_{-1}^{1} & {\left[G_{r z}^{r}(t, s)+f G_{z z}^{r}(t, s)\right] B_{r}(s) \mathrm{d} s } \\
& -\frac{2}{d}\left[\tilde{\sigma}_{r z}(t)+f \tilde{\sigma}_{z z}(t)\right] \quad-1 \leq t \leq 1 .
\end{aligned}
$$

Next, we need to consider the general form of the solution required. We note, first, that at $r=d$ the contact transitions smoothly from slip to stick. Thus, at this point $(t=1)$ the shear displacement gradient must be zero (both upper and lower surfaces displace the same amount tangentially), which means that the dislocation density must be bounded. At the other end of the integration interval $(r=0, t=-1)$, by symmetry, the tangential displacement gradient must be zero and, thus, the dislocation density is also bounded. Hence, we assume a fundamental function $B_{r}(s)$ as shown below, which leaves the unknown dislocation density being represented by the function $\phi_{r}(s)$ :

$$
B_{r}(s)=\phi_{r}(s) \sqrt{1-s^{2}}
$$

and eq. (20) becomes, in normalised form,

$$
\begin{gathered}
\sum_{i=1}^{N}\left\{\left[G_{r z}^{r}\left(t_{k}, s_{i}\right)+f G_{z z}^{r}\left(t_{k}, s_{i}\right)\right] W_{i} \phi_{r}\left(s_{i}\right)\right\} \\
=-\frac{2}{\pi d}\left[\tilde{\sigma}_{r z}(r, z)\left(t_{k}\right)+f \tilde{\sigma}_{z z}(r, z)\left(t_{k}\right)\right] \\
k=1, \ldots, N+1
\end{gathered}
$$

where the integration points $s_{i}$, collocation points $t_{k}$ and weights $W_{i}$ for the quadrature are given as [15]

$$
\begin{array}{rlrl}
s_{i} & =\cos \left(\pi \frac{i}{N+1}\right) & i=1, \ldots, N \\
t_{k}=\cos \left(\frac{\pi}{2} \frac{2 k-1}{(N+1)}\right) & i=1, \ldots, N+1 \\
W_{i}=\frac{1-s_{i}^{2}}{2(N+1)} &
\end{array}
$$

From eq. (22), we have a set of $N+1$ equations for $N+1$ unknowns. These are the $N$ values of $\phi_{r}\left(s_{i}\right)$ and the stick point $d$. Once $\phi_{z}$ is known, the stresses at a point $(r, z)$ can be found as

$$
\begin{gathered}
\sigma_{i z}(r, z)=\tilde{\sigma}_{i z}(r, z)+\int_{0}^{d} G_{i z}^{r}(r, z, \xi) B_{r}(\xi) \mathrm{d} \xi \\
i=r, z .
\end{gathered}
$$

\subsection{Case III}

The problem we look at now is when $b / a$ is large and $f$ is small, which allows for the contact to have an open region extending from the origin to a radius $r=c$ and a slip region from $r=c$ to $r=d$. Both the closure point $c$ and stick point $d$ are outputs of the problem (self-determining points).

In the open region, we require the surfaces to be tractionfree

$$
N(r)=0, \quad S(r)=0, \quad 0 \leq r \leq c
$$

whereas, in the closed, slipping region, the shearing traction is again limited by friction: 


$$
N(r)<0 \quad S(r)=-f N(r) \quad c \leq r \leq d .
$$

The three sets of conditions in eqs. (27) and (28) may be combined into two by making use of the Heaviside step function, $H(\cdot)$, giving

$$
\begin{aligned}
N(r) & =0 & & 0 \leq r \leq c \\
S(r)+f H(r-c) N(r) & =0 & & 0 \leq r \leq d .
\end{aligned}
$$

The climb dislocations are non-zero only over the interval $0 \leq r \leq c$, whereas the glide dislocations must be distributed over the whole interval $0 \leq r \leq d$. Applying eqs. (15) and (16) to eqs. (29) and (30), gives

$$
\begin{aligned}
& \int_{0}^{d} G_{z z}^{r}(r, \xi) B_{r}(\xi) \mathrm{d} \xi+\int_{0}^{c} G_{z z}^{z}(r, \xi) B_{z}(\xi) \mathrm{d} \xi \\
& =-\tilde{\sigma}_{z z}(r) \quad 0 \leq r \leq c \\
& \int_{0}^{d}\left[G_{r z}^{r}(r, \xi)+f H(r-c) G_{z z}^{r}(r, \xi)\right] B_{r}(\xi) \mathrm{d} \xi+ \\
& \int_{0}^{c}\left[G_{r z}^{z}(r, \xi)+f H(r-c) G_{z z}^{z}(r, \xi)\right] B_{z}(\xi) \mathrm{d} \xi \\
& =-\left[\tilde{\sigma}_{r z}(r)+f H(r-c) \tilde{\sigma}_{z z}(r)\right] \quad 0 \leq r \leq d .
\end{aligned}
$$

Since there are two regions of imposition for the integral equations, two sets of quadrature points are needed. Again, the equation must be solved numerically, using Gauss-Chebyshev quadrature [15]. Putting them in standard form over the intervals $[-1,1]$ using the substitutions

$$
\begin{array}{lll}
u=\frac{2 \xi}{c}-1, & v=\frac{2 r}{c}-1 & 0 \leq r, \xi \leq c \\
s=\frac{2 \xi}{d}-1, & t=\frac{2 r}{d}-1 & 0 \leq r, \xi \leq d
\end{array}
$$

gives

$$
\begin{aligned}
& \int_{-1}^{1} G_{z z}^{r}(v, s) B_{r}(s) d \mathrm{~d} s+\int_{-1}^{1} G_{z z}^{z}(v, u) B_{z}(u) c \mathrm{~d} u \\
& =-\tilde{\sigma}_{z z}(v) \quad-1 \leq v \leq 1 \\
& \int_{-1}^{1} B_{r}(s)\left[G_{r z}^{r}(t, s)+f H(\gamma) G_{z z}^{r}(t, s)\right] d \mathrm{~d} s+ \\
& \int_{-1}^{1} B_{z}(u)\left[G_{r z}^{z}(t, u)+f H(\gamma) G_{z z}^{z}(t, u)\right] c \mathrm{~d} u \\
& =-\left[\tilde{\sigma}_{r z}(t)+f H(\gamma) \tilde{\sigma}_{z z}(t)\right]-1 \leq t \leq 1
\end{aligned}
$$

where $\gamma=(t+1) d /(2 c)-1$.

For the general form of the solution, both the climb and glide dislocations must be bounded at both ends of the interval. Thus, we choose

$$
\begin{gathered}
B_{r}(s)=\phi_{r}(s) \sqrt{1-s^{2}} \\
B_{z}(u)=\phi_{z}(u) \sqrt{1-u^{2}}
\end{gathered}
$$

and eqs. (35) and (36) become, in normalised form,

$$
\begin{aligned}
& \sum_{i=1}^{N}\left\{W_{i} d \phi_{r}\left(s_{i}\right) G_{z z}^{r}\left(v_{k}, s_{i}\right)+X_{i} c \phi_{z}\left(u_{i}\right) G_{z z}^{z}\left(v_{k}, u_{i}\right)\right\} \\
& =-\frac{2}{\pi} \tilde{\sigma}_{z z}\left(v_{k}\right) \quad k=1, \ldots, N+1 \\
& \sum_{i=1}^{N}\left\{\left[G_{r z}^{r}\left(t_{k}, s_{i}\right)+f H\left(\gamma_{k}\right) G_{z z}^{r}\left(t_{k}, s_{i}\right)\right] W_{i} d \phi_{r}\left(s_{i}\right)\right\}+ \\
& \sum_{i=1}^{N}\left\{\left[G_{r z}^{z}\left(t_{k}, u_{i}\right)+f H\left(\gamma_{k}\right) G_{z z}^{z}\left(t_{k}, u_{i}\right)\right] X_{i} c \phi_{z}\left(u_{i}\right)\right\} \\
& =-\frac{2}{\pi}\left[\tilde{\sigma}_{r z}\left(t_{k}\right)+f H\left(\gamma_{k}\right) \tilde{\sigma}_{z z}\left(t_{k}\right)\right] k=1, \ldots, N+1
\end{aligned}
$$

where $\gamma_{k}=\left(t_{k}+1\right) d /(2 c)-1$. Since both dislocations have the same behaviour, $s_{i}=u_{i}, t_{k}=v_{k}$ and $W_{i}=$ $X_{i}$. Furthermore, they are the same as those presented in Section 3.1 and are given in eqs. (23) to (25).

Equations (39) and (40) form a system of $2 N+2$ equations and $2 N+2$ unknowns. These are the $N$ values of $\phi_{r}\left(s_{i}\right), N$ values of $\phi_{z}\left(u_{i}\right)$, the closure point $c$ and the stick point $d$. Once $\phi_{r}$ and $\phi_{z}$ are known, the stresses at a point $(r, z)$ can be found as

$$
\begin{aligned}
\sigma_{i z}(r, z)= & \tilde{\sigma}_{i z}(r, z)+\int_{0}^{d} G_{i z}^{r}(r, z, \xi) B_{r}(\xi) \mathrm{d} \xi+ \\
& \int_{0}^{c} G_{i z}^{z}(r, z, \xi) B_{z}(\xi) \mathrm{d} \xi \quad i=r, z .
\end{aligned}
$$

\section{Results}

The two problems relating to cases $I I$ and $I I I$ were coded up using the numerical processor MATLAB. Setting $N$ to 80 makes the solution converge for both cases, i.e. the changes in the closure point $c$ and stick point $d$, as well as changes in stress, are negligible when $N$ is increased beyond 80. All the results presented in this paper are for $\nu=0.3$. In problems where a length dimension is an unknown of the problem ( $d$ in case $I I$ and both $c, d$ in case $I I I)$, there are additional collocation equations which need to be satisfied and which enable the values of $c / a$ and $d / a$ to be found. In practice, we guess values of the lengths to be found and omit the last equations from the $N+1$ generated. The column vector of $\phi$ is found. The omitted equations are then evaluated and the lengths needed are adjusted to minimise the residues. 


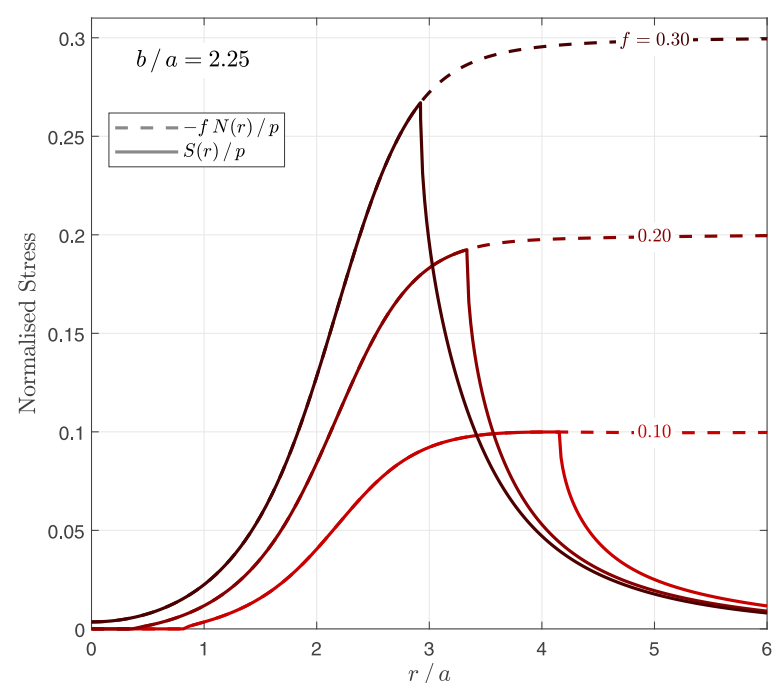

(a)

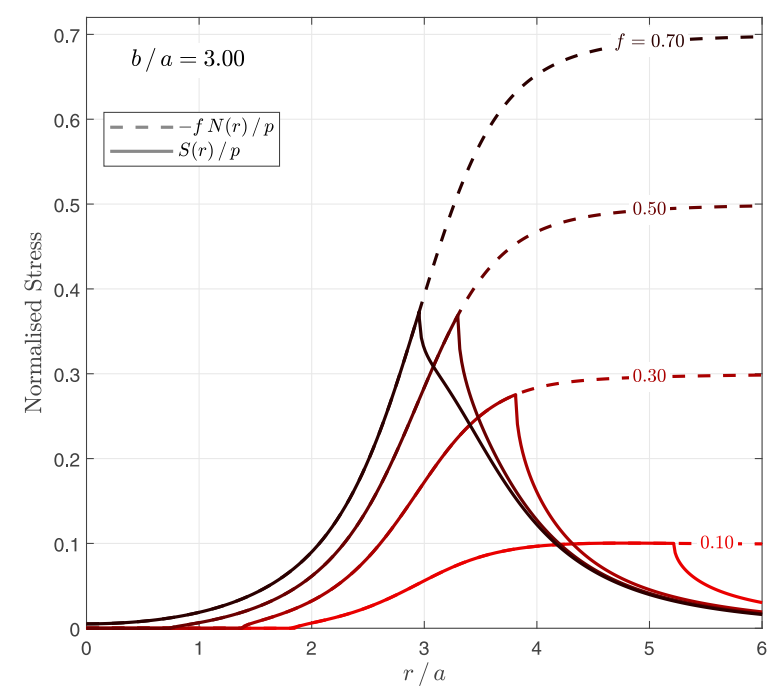

(b)

Figure 3: Normalised tractions in the contact interface. (a) $b / a=$ 2.25. (b) $b / a=3.00$.

Figure 3 shows the contact pressure and shear stress normalised by the applied pressure $p$ for $b / a=2.25$ and 3.00. We notice that the stresses are proportional to the applied pressure, i.e. for a given $b / a$ doubling the applied pressure $p$ would result in the stresses being doubled, such that the normalised traction distributions remain constant. Since the distributions do not change, the points of stick and closure do not change as well. The contact size is, therefore, independent of $p$ and varies only with the coefficient of friction and the normalised radius of the unloaded disk $b / a$. For any increment of load the contact 'snaps' from the undeformed unloaded condition to the deformed configuration, which means that, as ex-

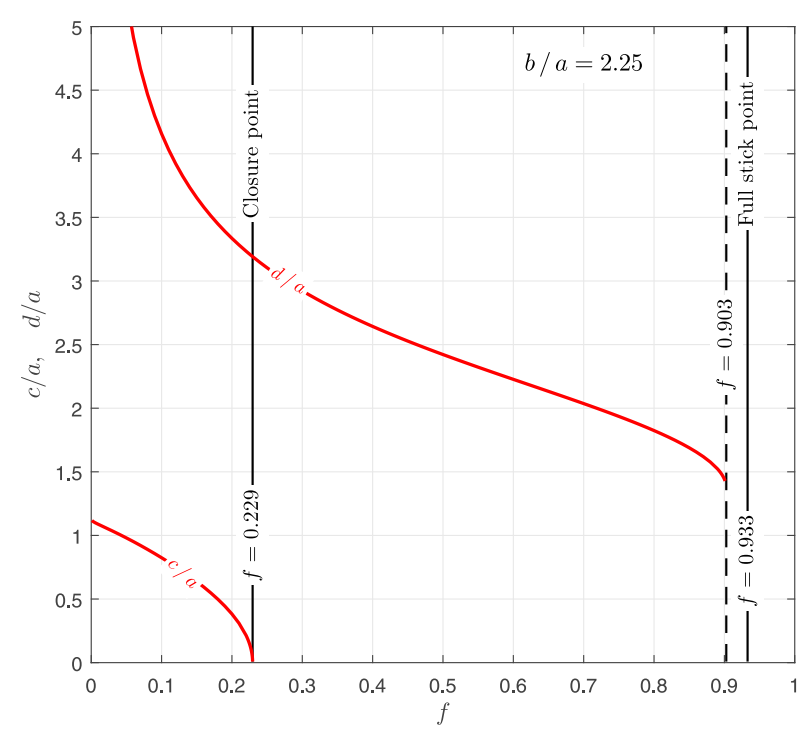

Figure 4: Normalised closure point $c / a$ and stick point $d / a$ as a function of the coefficient of friction for $b / a=2.25$.

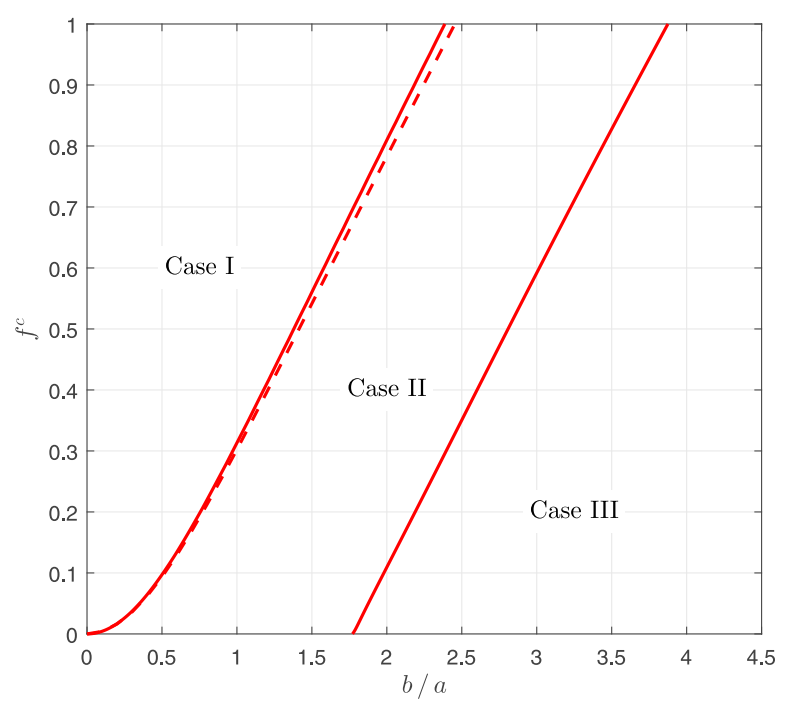

Figure 5: Transition between cases.

pected, the contact area changes discontinuously from an infinite size when unloaded to a finite size upon applying any increment of load $[1,2,16]$.

Consider, now, the values of normalised stick point $d / a$ and closure point $c / a$ as a function of the coefficient of friction for $b / a=2.25$ (Figure 4). For $0 \leq f<0.229$, the contact is in Case $I I I$. As $f$ increases, the opening and slip regions decrease, but the contact remains in Case $I I I$ (there is an open region). When $f$ reaches $0.229, c / a=$ 0 and the contact closes. For $0.229<f<0.903$, the contact is in Case $I I$ and as $f$ increases the radius of the slip zone decreases. For $0.903<f<0.933$, there are no solutions for $d / a$ in Case $I I$. This region characterizes an intermediate state between Cases $I I$ and $I$ where the 


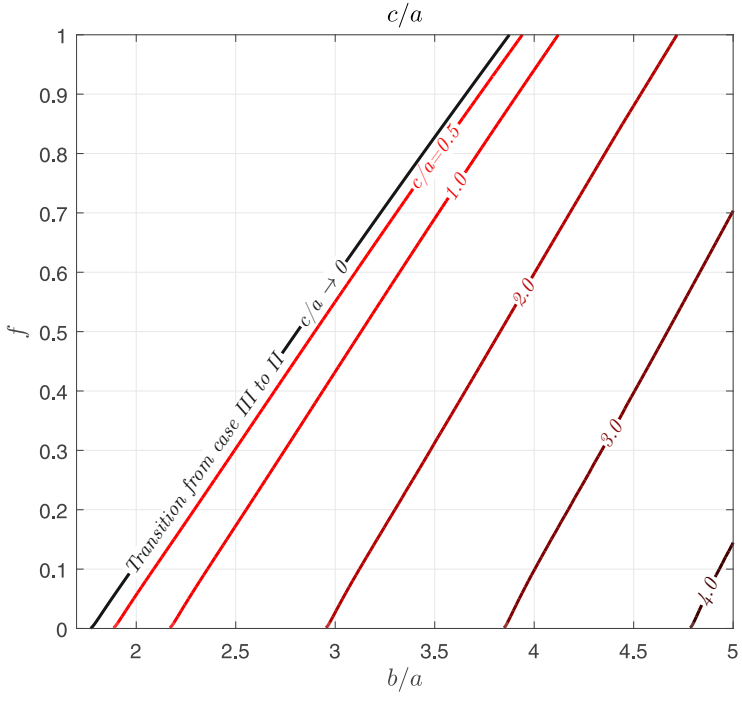

Figure 6: Normalised point of closure $c / a$.

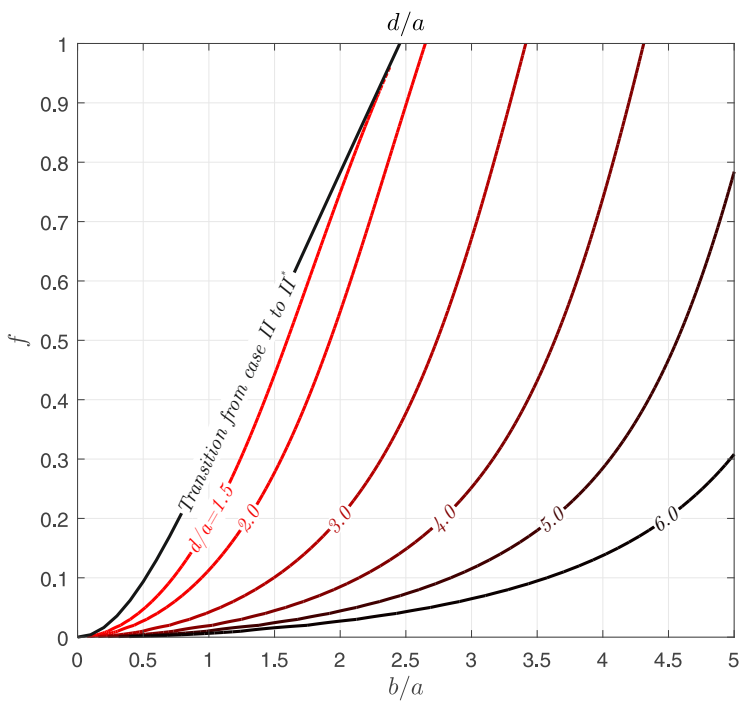

Figure 7: Normalised point of stick $d / a$.

contact has a finite region of slip inside the contact (Case $\left.I I^{*}\right)$. Because this case represents only a small portion of the results, its solution was not found. The line $f=0.933$ represents the minimum coefficient of friction that results in the contact being fully stuck. For $f>0.933$, the contact is in Case $I$ (fully closed and stuck).

This same procedure can be used to find the transition values between cases for pairs of $b / a$ and $f$ (Figure 5). From Cases $I I$ to $I I I$, the transition is characterized by contact opening at the origin. Therefore, we look for the value of $b / a$ that results in $N(0)=0$ for a given $f$ (rightmost solid line in Figure 5). In Case $I$, the transition is given by the minimum coefficient of friction required to guarantee no slip for each $b / a$ (leftmost solid line in Fig- ure 5). Finally, the dashed line represents the limit in solution for Case $I I$. The region inside the solid and dashed lines between Cases $I$ and $I I$ represents the intermediate Case $I I^{*}$.

Figures 6 and 7 show the contours of normalised point of closure $c / a$ and point of stick $d / a$ as functions of $f$ and $b / a$. It may be noted that the normalised closure point is always smaller than the unloaded radius $b / a$, i.e. contact opening never extends into the loaded region. Also, $c / a$ decreases as the coefficient of friction increases and/or $b / a$ becomes smaller. The contact closes at the line of $c / a=$ 0 (black line in Figure 6 ). The point of stick $d / a$ also decreases when $f$ increases and/or $b / a$ becomes smaller.

If there is no friction, from Figure 5 , the contact is in case $I I$ if $b / a<1.771$ and in Case $I I I$ otherwise. In Case $I I$, the point of stick is finite and increases as $b / a$ increases (Figure 7). In Case $I I I$, the point of closure is finite and increases as $b / a$ increases (Figure 6 ) but $d / a \rightarrow \infty$, which means that the contact would be sliding and not in elastic equilibrium (Figures 4 and 7 ).

\section{Conclusions}

The contact properties and tractions were obtained for a receding contact between a layer subjected to axisymmetric 'external' semi-infinite pressure and an elastically similar half-space. It was shown that the problem is fully characterised by two variables, the coefficient of friction between interfaces $f$ and the normalised length of the unloaded radius $b / a$. Depending on the relationship between $f$ and $b / a$, the contact may be fully closed and stuck (Case $I$ ), closed and partially slipping from the axis of symmetry (Case $I I$ ), closed and partially slipping inside the contact interface (Case $I I^{*}$ ), or partially open and slipping (Case $I I I)$. The curves that characterise the transition between cases were also found.

In addition, it was shown that, as expected, the interfacial tractions are proportional to the applied load while the contact area is independent of it and changes discontinuously from the unloaded to loaded configuration with the application of an incremental load

Finally, in Case $I I I$ it was found that contact opening never penetrates into the loaded regions, as $d / a$ is always smaller than the unloaded radius $b / a$.

\section{Acknowledgements}

J. L. gratefully acknowledges the financial support of Christ Church Oxford, Rolls Royce PLC and Coordenação de Aperfeiçoamento de Pessoal de Nível Superior - CAPES.

\section{References}

[1] J. Dundurs, Properties of elastic bodies in contact, The Mechanics of the Contact between Deformable bodies (1975) 54-66.

[2] J. Barber, Contact Mechanics, Solid Mechanics and Its Applications, Springer International Publishing, 2018. 
[3] T. Chaise, R. Paynter, D. Hills, Contact analysis of a semiinfinite strip pressed onto a half plane by a line force, International Journal of Mechanical Sciences 81 (2014) 60-64.

[4] K. Parel, D. Hills, Frictional receding contact analysis of a layer on a half-plane subjected to semi-infinite surface pressure, International Journal of Mechanical Sciences 108 (2016) 137-143.

[5] L. Keer, J. Dundurs, K. Tsai, Problems involving a receding contact between a layer and a half space, Journal of Applied Mechanics 39 (4) (1972) 1115-1120.

[6] K. Tsai, J. Dundurs, L. Keer, Contact between an elastic layer with a slightly curved bottom and a substrate, Journal of Applied Mechanics, Transactions ASME 39 (3) (1972) 821-823.

[7] K. Tsai, J. Dundurs, L. Keer, Elastic layer pressed against a half space, Journal of Applied Mechanics 41 (3) (1974) 703-707.

[8] K.-i. Terazawa, On the elastic equilibrium of a semi-infinite solid under given boundary conditions, Journal of the College of Science (1916) 14-24.

[9] A. E. H. Love, The stress produced in a semi-infinite solid by pressure on part of the boundary, Philosophical Transactions of the Royal Society of London. Series A, Containing Papers of a Mathematical or Physical Character 228 (659-669) (1929) $377-420$.

[10] M. T. Hanson, I. W. Puja, Love's circular patch problem revisited: Closed form solutions for transverse isotropy and shear loading, Quarterly of applied mathematics 54 (2) (1996) 359384 .

[11] D. Hills, P. Kelly, D. Dai, A. Korsunsky, Solution of crack problems: the distributed dislocation technique, Vol. 44 of Solid Mechanics and Its Applications, Springer Netherlands, Dordrecht, 2013.

[12] R. Paynter, D. Hills, The effect of path cut on Somigliana ring dislocations in a half-space, International Journal of Solids and Structures 46 (2) (2009) 412-432.

[13] R. Paynter, D. Hills, A. Korsunsky, The effect of path cut on Somigliana ring dislocation elastic fields, International Journal of Solids and Structures 44 (2) (2007) 6653-6677.

[14] J. Lopes, D. Hills, Ring cracks at the surface of a half-space, Engineering Fracture Mechanics 194 (2018) 105-116

[15] F. Erdogan, G. D. Gupta, T. Cook, Numerical solution of singular integral equations, in: Methods of analysis and solutions of crack problems, 1973, pp. 368-425.

[16] J. Dundurs, M. Stippes, Role of elastic constants in certain contact problems, Journal of Applied Mechanics 37 (4) (1970) 965-970.

[17] G. Eason, B. Noble, I. N. Sneddon, On certain integrals of Lipschitz-Hankel type involving products of Bessel functions, Philosophical Transactions of the Royal Society of London A: Mathematical, Physical and Engineering Sciences 247 (935) (1955) 529-551.

[18] E. Gordeliy, E. Detournay, Displacement discontinuity method for modeling axisymmetric cracks in an elastic half-space, International Journal of Solids and Structures 48 (19) (2011) 2614 2629 .

\section{Appendix A. State of stress induced by circular edge dislocation loops}

\section{Appendix A.1. Axial dislocation}

Consider a climb axial dislocation loop of radius $a$ put at a depth $d$ and being observed at a position $(r, z)$ in a cylindrical coordinate system, with a Burgers vector component $b_{z}$. The stress fields at a position $(r, z)$ are given by

$$
\sigma_{i z}^{z}(r, z)=G_{i z}^{z}(r, z, d) b_{z}(a) \quad i=r, z
$$

The influence functions $G_{i z}^{z}(\rho, \zeta, \delta)(i=r, z)$ for the glide dislocation in a half-space are given as [12]:

$$
\begin{aligned}
G_{z z}^{z}(\rho, \zeta, \delta)= & \frac{2 \mu}{a(\kappa+1)}\left[-J_{1,0 ; 1}+I_{1,0 ; 1}-\right. \\
& (\zeta-\delta) J_{1,0 ; 2}-(\zeta+\delta) I_{1,0 ; 2}+ \\
& \left.2 \zeta \delta I_{1,0 ; 3}\right] \\
G_{r z}^{z}(\rho, \zeta, \delta)= & \frac{2 \mu}{a(\kappa+1)}\left[-(\zeta-\delta) J_{1,1 ; 2}+\right. \\
& \left.(\zeta-\delta) I_{1,1 ; 2}-2 \zeta \delta I_{1,1 ; 3}\right]
\end{aligned}
$$

where $\rho, \zeta$ and $\delta$ are the normalised coordinates, given as

$$
\rho=r / a, \quad \zeta=z / a, \quad \delta=d / a,
$$

$\mu$ is the modulus of rigidity and $\kappa$ is the Kolosov's constant.

\section{Appendix A.2. Radial dislocation}

The radial dislocation is not of Volterra kind and, thus, is path-cut dependent. In this paper, an 'inside disk' path cut is used [12]. This path cut can be formed by inserting a disk of material at a depth $z=d$, from $r=0$ to $r=a$, displacing the material by the same amount $b_{r}$ (thickness of the disk). The stress fields at a position $(r, z)$ are given by

$$
\sigma_{i z}^{r}(r, z)=G_{i z}^{r}(r, z, d) b_{r}(a) \quad i=r, z .
$$

The influence functions $G_{i z}^{r}(\rho, \zeta, \delta)(i=r, z)$ for the glide dislocation in a half-space are given as [12]:

$$
\begin{aligned}
G_{z z}^{r}(\rho, \zeta, \delta)= & \frac{2 \mu}{a(\kappa+1)}\left[-(\zeta-\delta) J_{2,0 ; 2}+(\zeta-\delta) I_{2,0 ; 2}-\right. \\
& \left.2 \zeta \delta I_{2,0 ; 3}\right]
\end{aligned}
$$

$$
\begin{aligned}
G_{r z}^{r}(\rho, \zeta, \delta)= & \frac{2 \mu}{a(\kappa+1)}\left[-J_{2,1 ; 1}+I_{2,1 ; 1}+\right. \\
& (\zeta-\delta) J_{2,1 ; 2}+(\zeta+\delta) I_{2,1 ; 2}+ \\
& \left.2 \zeta \delta I_{2,1 ; 3}\right]
\end{aligned}
$$




\section{Appendix A.3. Lipschitz-Hankel integrals}

In the influence functions, the terms $J_{n, p ; q}$ and $I_{n, p ; q}$ represent Lipschitz-Hankel integrals. The standard definition for these functions is as an integral of the product of Bessel functions $\left(J_{i}(\cdot)\right)$, an exponential term and a power term. Using normalised coordinate variables, it is given as [17]

$$
P_{\mu, \nu ; \lambda}(\rho, \zeta)=\int_{0}^{\infty} J_{\mu}(t) J_{\nu}(\rho t) e^{-\zeta t} t^{\lambda} \mathrm{d} t .
$$

In the kernels, the follow definition is applied:

$$
\begin{aligned}
J_{n, p ; q} & =P_{n, p ; q}(\rho, \zeta-\delta) \\
I_{n, p ; q} & =P_{n, p ; q}(\rho,-\zeta-\delta)
\end{aligned}
$$

The Lipschitz-Hankel integrals needed in the kernels are given by Paynter et al. [13, 12] (see also [18]). 